\title{
Influence of Deep Learning Image Reconstruction (DLIR) and Adaptive Statistical Iterative Reconstruction-V (ASIR-V) on Coronary Artery Calcium Quantification
}

\author{
Yiran Wang ( $13298363780 @ 163 . c o m)$ \\ The First Affiliated Hospital of Zhengzhou University \\ Hefeng Zhan \\ The First Affiliated Hospital of Zhengzhou University

\section{Wenjie Wu} \\ The First Affiliated Hospital of Zhengzhou University \\ Jie Liu \\ The First Affiliated Hospital of Zhengzhou University \\ Jianbo Gao \\ The First Affiliated Hospital of Zhengzhou University \\ Ying Guo \\ GE Company,Beijing

\section{Yonggao Zhang}

The First Affiliated Hospital of Zhengzhou University https://orcid.org/0000-0001-6027-4650

\section{Research Article}

Keywords: Coronary artery disease, Calcium scoring, Deep Learning Image Reconstruction, Adaptive Statistical Iterative Reconstruction-V, Risk stratification

Posted Date: July 30th, 2021

DOl: https://doi.org/10.21203/rs.3.rs-758809/v1

License: (9) This work is licensed under a Creative Commons Attribution 4.0 International License. Read Full License 


\title{
Influence of Deep Learning Image Reconstruction (DLIR) and Adaptive Statistical Iterative Reconstruction-V (ASIR-V) on Coronary Artery Calcium Quantification
}

\author{
Yiran Wang ${ }^{a}$, Hefeng Zhan ${ }^{a}$, Wenjie Wu ${ }^{a}$, Jie Liu ${ }^{a}$, Jianbo Gao ${ }^{a}$, Ying Guo $^{\text {b }}$ and Yonggao \\ Zhang a, * \\ ${ }^{a}$ Department of Radiology, the First Affiliated Hospital of Zhengzhou University, Zhengzhou, China \\ ${ }^{b}$ GE Company, Beijing, China
}

*Corresponding author: Yonggao Zhang, PhD, Department of Radiology, the First Affiliated Hospital of Zhengzhou University, No.1 Jianshe Road East, Zhengzhou 450052, China. Tel: +86 037166913114 ; Fax: +86 037166913069; E-mail: zyg01578@126.com 


\section{Abstract}

Deep learning image reconstruction (DLIR) and Adaptive Statistical Iterative Reconstruction-V (ASIR-V) has been used for cardiac computed tomography imaging. However, DLIR and ASIR-V may influence the quantification of coronary artery calcification. This study aimed to investigate the effects of DLIR and ASIR-V on coronary calcium quantification compared to traditional filtered back projection (FBP). CT images of 96 patients were reconstructed by FBP, ASIR-V 50\%, and three levels of DLIR (low [L], medium [M], and high $[\mathrm{H}]$, respectively). Image noise decreased significantly with ASIR-V 50\% and increasing DLIR levels from L to $\mathrm{H}$ in comparison with FBP (all $\mathrm{P}<0.001$ ). There is a significantly decline with ASIR-V $50 \%$ and incremental DLIR levels in Agatston calcium score, volume score and mass score as compared to FBP (all P < 0.001). For all CAC score risk categories, Severity classification shows no significant differences among five reconstructions (all $\mathrm{P}>0.05$ ). DLIR-L has the minimal effect on coronary calcium quantification as compared to ASIR-V and DLIR at medium and high levels. it may be considered as an alternative to FBP for routine clinical use.

Key Words Coronary artery disease, Calcium scoring, Deep Learning Image Reconstruction, Adaptive Statistical Iterative Reconstruction-V, Risk stratification

\section{Declarations}

\section{Funding}

This research did not receive any specific grant from funding agencies in the public, commercial, or not-forprofit sectors.

\section{Conflicts of interest/Competing interests}

The authors declare that they have no conflict of interest.

\section{Availability of data and material}

The datasets used or analyzed during the current study are available from the corresponding author on reasonable request.

\section{Code availability}

Not applicable

\section{Authors' contributions}

All authors contributed to the study conception and design. Material preparation, data collection and analysis were performed by Yiran Wang, Hefeng Zhan and Wenjie Wu. The first draft of the manuscript was written by Yiran Wang and all authors commented on previous versions of the manuscript. All authors read and approved the final manuscript.

\section{Ethics approval}


The questionnaire and methodology for this study was approved by the Human Research Ethics committee of the University of the First Affiliated Hospital of Zhengzhou University (Ethics approval number: 2021-KY0043-002).

\section{Consent to participate}

Informed consent was obtained from all individual participants included in the study.

\section{Consent for publication}

Patients signed informed consent regarding publishing their data and photographs.

\section{Acknowledgments}

We gratefully acknowledge Dr. Jie Liu from Department of Radiology, the First Affiliated Hospital of Zhengzhou University for his technical support in this study. 


\section{Introduction}

Coronary artery calcium (CAC) quantified on non-contrast CT examinations is a robust risk stratification tool for cardiovascular risk stratification, it provides high predictive value for incident cardiovascular events in asymptomatic patients [1-3], particularly those at intermediate cardiovascular risk. Calcification score scanning has become a common method for monitoring of coronary atherosclerotic plaque progression, which is prevalent used in patients who do not suit for enhanced examinations.

With innovations in CT techniques, reconstruction algorithms by various vendors and lowering tube voltage / tube current has been extensively investigated in CT coronary angiography [4-5]. however, CAC acquisition protocol still needs to be preset in $120 \mathrm{kVp}$ and reconstructed by filter back projection (FBP) [6], which lead to a relatively high level of image noise and radiation dose. There has been increased concerns on medical-related radiation exposure and its relevant radiation induced cancer risk [7-8]. iterative reconstruction (IR) [9-11] available commercially from various vendors allows for radiation dose savings while maintaining adequate image quality, but the IR algorithm may change the noise amplitude and texture features of the image, particularly at high strengths of iterative reconstructions [12-13].

In recent years, applications of artificial intelligence [14-15] have already shown significant promise in cardiovascular imaging. a deep learning-based image reconstruction [16] (DLIR, TrueFidelity, GE Healthcare) uses a convolutional neural network $(\mathrm{CNN})$ shows great potential that improves image reconstruction in $\mathrm{CT}$, which has been verified by some researchers in phantom [17] and clinical studies [18-19] to reduce image noise. Simultaneously, the image texture of DLIR is similar to that of high-dose filtered back projection (FBP) [17].

However, the influence of the new reconstruction algorithms in quantification of calcification score remains unclear. Accordingly, the current study aims to investigate the effects of DLIR and ASIR-V on quantitative evaluation of coronary calcification and subsequent cardiovascular risk stratification compared with the traditional FBP.

\section{Methods}

\subsection{Study population}

This study protocol was approved by local ethics committee (2021-KY-0043-002), all patients have signed informed consent forms. 96 patients referred for coronary CT angiography due to suspected coronary artery disease were enrolled between February 2021 and April 2021. All patients underwent ECG-gated non-contrast CT for CAC scans before CTA examination. Patients with the following characteristics were excluded: contrast media allergy, chronic kidney disease (serum creatinine $>1.5 \mathrm{mg} / \mathrm{dL}$ or glomerular filtration rate $<45$ $\mathrm{mL} / \mathrm{min} / 1.73 \mathrm{~m}^{2}$ ), unable to hold the breath for $15 \mathrm{~s}$, pregnancy, atrial fibrillation, body mass index $(\mathrm{BMI})>40$ $\mathrm{kg} / \mathrm{m}^{2}$, coronary stent implantation and previous coronary artery bypass grafting.

\subsection{CT acquisition}

All checks are performed with a 256-slice CT scanner (Revolution CT, GE Healthcare, Waukesha, WI). patients with a resting heart rate $>70$ beats/min received oral beta-blockers unless contraindicated. Calcium scoring scan was performed using a prospective electrocardiogram (ECG)-gating, triggering at $75 \%$ of the R-R interval with the following acquisition parameters: $120 \mathrm{kV}$ tube voltage, automatic tube current modulation with a range of 100-720 mA, $12 \mathrm{HU}$ noise index, $256 \times 0.625 \mathrm{~mm}$ collimation, $280 \mathrm{~ms}$ gantry rotation time, 140 or $160 \mathrm{~mm}$ scan coverage, and display field of view $25 \mathrm{~mm}$.

The volume CT dose index $\left(\mathrm{CTDI}_{\mathrm{vol}}\right)$ and dose length product (DLP) were recorded on the dose report. The 
effective radiation dose (ED) was estimated by multiplied the DLP by a body region-specific conversion coefficient $\left(\mathrm{k}=0.014 \mathrm{mSv} \times \mathrm{mGy}^{-1} \times \mathrm{cm}^{-1}\right)$.

\subsection{Image reconstruction and analysis}

Calcium scoring scanning images were reconstructed by standard FBP, ASIR-V at a level of $50 \%$, and three strength levels of DLIR (low [L], medium [M], and high [H], respectively) with a section thickness of $2.5 \mathrm{~mm}$ and without padding.

Objective image quality parameters were carried out on offline workstation (Advantage Workstation Version 4.7, GE Healthcare). The CT value and image noise were measured as the mean signal value in Hounsfield units (HU) and standard deviation (noise) in the aortic root and the left ventricle using a region of interest (ROI) of $0.5 \mathrm{~cm}^{2}$. Compare Viewer (GE Healthcare) was used to ensure that all images were at the same level. signalto-noise ratio (SNR) was calculated as the signal divided by the noise.

A semiautomatic commercially available software (SmartScore 4.0, GE Healthcare) was used for detection of calcified lesions. All pixels with a density equal to or greater than a fixed threshold (130 HU) having an area > $1 \mathrm{~mm}^{2}$ were marked automatically. then the user identified calcified lesions manually based on their anatomical location. the Agatston score, lesion volume $\left(\mathrm{mm}^{3}\right)$ and mass $(\mathrm{mg})$ were automatically calculated finally. Patients were classified into five CAC score risk categories according to their Agatston score determined by FBP: 0, no risk, 1-10, low, 11-100, moderate, 101-400, high and > 400, very high risk, respectively. According to the Multi-Ethnic Study of Atherosclerosis (MESA) study [20], patients were grouped with similar age, gender and race into five Agatston score percentile-based risk categories $(0-25,26-$ $50,51-75,76-90$, and $>90$ ). the patient's number of who switching to another risk stratification with the use of ASIR-V and DLIR with reference to FBP been recorded.

\subsection{Statistics}

Statistical analyses were performed using dedicated statistical software (SPSS 22.0; SPSS, Chicago, IL). The data were tested for normal distribution by Shapiro-Wilk test. Continuous variables were expressed as mean \pm standard deviation or median and interquartile range (IQR), while categorical variables as percentages and counts. McNemar's test were used to assess the Agatston score classes and the Agatston score percentile-based risk categories. Noise measurements, and CAC variables were compared by using Wilcoxon signed-rank test as well as Boneferroni's method for multiple comparisons. The agreement of Agatston scores between FBP and different reconstructions were presented using Bland-Altman plots. A p value of 0.05 or less was considered significant.

\section{Results}

\subsection{Patient characteristics}

The baseline characteristics and radiological parameters of included patients are listed in Table 1. the study population consisted of 96 patients in whom $60 \%$ was male, with a mean age of $65.3 \pm 10.2$ years, and a mean body mass index (BMI) of $23.9 \pm 3.3 \mathrm{~kg} / \mathrm{m}^{2}$, the mean effective patient dose was $0.93 \pm 0.15 \mathrm{mSv}$.

\subsection{Image noise}

The mean noise decreased $26.7 \pm 3.4 \%, 27.6 \pm 8.3 \%, 37.3 \pm 7.2 \%$, and $46.4 \pm 5.4 \%$ in the aortic root and $25.9 \pm 3.2 \%, 23.7 \pm 9.2 \%, 34.6 \pm 7.7 \%$, and $44.9 \pm 5.7 \%$ in the left ventricular cavity for reconstructions 
with ASIR-V 50\% and three levels of DLIR (DLIR-L, M, and H, respectively) in comparison to FBP (all $\mathrm{P}<$ 0.05). $\mathrm{P}<0.05$ of DLIR-M and DLIR-H compared to ASIR-V 50\%. there were no significant differences for DLIR-L in comparison with ASIR-V 50\% $(\mathrm{p}>0.05)$. there are significant differences among three levels of DLIR (all $\mathrm{p}<0.05$ ). with DLIR-H giving the greatest noise reduction (Fig. 2). the data of mean CT value, SNR in aortic root and left ventricular cavity are shown in Online Resource 1. the mean CT value in aortic root and left ventricular cavity keep constant among groups $(p>0.05)$. As a result, there was a significant rise in SNR with increasing strengths of DLIR in the aortic root from $2.3 \pm 0.5$ to $4.3 \pm 1.1$ and the left ventricular cavity from $2.1 \pm 0.6$ to $3.7 \pm 1.2$ for FBP and DLIR level $\mathrm{H}$ (all $\mathrm{p}<0.05$ ). there was also a significant rise in SNR for DLIR-M and DLIR-H compared to ASIR-V 50\%, as well as ASIR-V 50\% compared to FBP ( $\mathrm{p}<$ 0.05 , in both aortic root and left ventricular cavity). Table 2 shows the data of relative differences (expressed in mean percentages $\pm \mathrm{SD}$ ) between the targeted and the referenced reconstruction groups.

\subsection{CAC Scoring}

As the patients with zero CAC scores in FBP remained unchanged with reconstruction by ASIR-V and DLIR. Thus, we included 89 patients with CAC scores of above one to compare Agatston scores, calcium volumes and CAC mass among the 5 reconstruction techniques. Data were shown in Table 3.

The median values of Agatston calcium scores were 199 (IQR 32-451), 188 (IQR 26-422), 196 (IQR 30-426), 192.0 (IQR 28 -419), and 185 (IQR 28 -408) for FBP, ASIR-V 50\%, DLIR-L, DLIR-M, and DLIR-H, respectively. Accordingly, the relative differences for ASIR-V 50\% and increasing DLIR levels compared to FBP were $5.5 \%, 1.5 \%, 3.5 \%$ and $7.0 \%$ (all $\mathrm{p}<0.001$ ). Agatston score in DLIR-L was bigger and in DLIR-H was smaller than ASIR-V (both $\mathrm{p}<0.001$ ). there was no significant difference for DLIR-M compared to ASIRV 50\% $(\mathrm{p}=0.23)$. there was significant difference in Agatston score among DLIR reconstructions (all $\mathrm{p}<$ 0.05). The median CAC volume from the ASIR-V and increasing DLIR levels groups were differed significantly than those from the FBP image series [99 (20.8, 179.5), $95(15,165), 95.5(15.8,173), 93(15$, $169.8) \mathrm{mm} 3$ vs. $\left.91(14,164.8) \mathrm{mm}^{3}\right]$. Post-hoc analysis showed significant difference among the 5 techniques (each $\mathrm{P}<0.05)$. The median values of mass scores were $32(7,63), 29(6,61), 31(6.5,63), 30(6.5,59)$, and 30 $(6.5,59) \mathrm{mg}$ for FBP, ASIR-V 50\%, DLIR-L, DLIR-M, and DLIR-H, respectively. Post-hoc analysis showed significant difference for ASIR-V and increasing DLIR levels compared to FBP (each P $<0.05$ ). there was no significant difference for DLIR-M and DLIR-H compared to ASIR-V 50\% $(\mathrm{p}=0.186,0.858)$. The mass score in DLIR-L was bigger than ASIR-V $(\mathrm{P}<0.05)$. However, there was no significant difference in mass score among DLIR reconstructions (all $\mathrm{p}>0.05$ ). The Bland-Altman plots of Agatston scores with the mean and limits of agreement for images reconstructed by ASIR-V and increasing DLIR levels compared to FBP are shown in Fig. 3.

For the risk classification analysis, in comparison with FBP, the numbers of patients with CAC scores of one or greater reassigned to another risk category with ASIR-V 50\% and increasing DLIR levels from L to $\mathrm{H}$ were 5 $(5.6 \%), 3(3.4 \%), 5(5.6 \%)$, and $5(5.6 \%)$, The detailed data is available in Online Resource 2. Severity classification shows no significant differences among reconstructions (all $p>0.05$ ). No patients moved two or more risk categories. Additionally, Agatston score, age, sex, and race adjusted CAC percentile risk categories among FBP, ASIR-V 50\% and different levels of DLIR did not differ significantly (all $\mathrm{p}>0.05$ ). 85 patients from 45 to 85 years old were included according to the MESA study. Compared to FBP, a change in risk category was observed in 4 (4.7\%), 3 (3.5\%), 4 (4.7\%), and 5 (5.9\%) patients when CAC scores were acquired from ASIR-V 50\% and DLIR levels L, M, and H, respectively (the data are shown in Online Resource 3). A patient of fifty-seven years decreased to zero Agatston score (no risk, $0-25$ th percentile) in groups reconstructed by ASIR-V 50\%, DLIR-M and DLIR-H. However, the Agatston score was 1 (low risk, 50th75th percentile) in FBP group. The patient numbers of zero Agatston score in DLIR-L group remain unchanged. 


\section{Discussion}

Our study demonstrated that, in comparison to the standard FBP algorithm, DLIR and ASIR-V reduce the Agatston score, calcium mass and calcium volume. Although the Agatston score decreased statistically significant with ASIR-V and increasing DLIR strength levels, there was no significant difference in the Agatston score-based cardiovascular risk stratification among the reconstruction techniques. DLIR-L has the least effect on coronary calcium quantification as compared to ASIR-V and DLIR at medium and high levels. Furthermore, we have demonstrated that DLIR and ASIR-V resulted in significant noise reduction, the signal keeps constant, thus improving the SNR.

It is reported that [21-24] the improvement of image quality (such as the reduction of image noise) usually leads to the reduction in the CAC score. however, the widespread existence of image noise may hinder the dependable quantification on CAC. Several studies have confirmed the incremental value of CAC score in improving cardiovascular risk stratification and treatment decisions, including downstream detection [4]. With the introduction of Coronary artery calcification data and reporting system (CAC-DRS) [25] as an expert consensus document of the Cardiovascular Computed Tomography Society, in order to make meaningful comparison during clinical follow-up, correct and reproducible CAC acquisition becoming more essential [26]. With the rapid development of multi-slice spiral CT technology, new reconstruction algorithms continue to appear. At present, reconstruction algorithms by various vendors have different effect on cardiovascular risk assessment. Kurata et al. [21] published results that the Agatston score with sinogram-affirmed iterative reconstruction algorithm decreased by $48 \%$ compared to FBP. Messeli et al. [22] also demonstrated a significant reduction of $39 \%, 41 \%$ and $40 \%$ of the Agatston, volume and mass score in high-level ADMIRE. while in our study, Agatston, volume and mass values decreased by $7 \%, 8.1 \%$, and $6.3 \%$ in high strength level DLIR as compared to FBP, respectively. Szilveszter et al. [23] used data from the cardiovascular screening program in asymptomatic people to assess the number of risk reclassification by virtue of iterative reconstruction technique, they noticed that the risk category derived from the CAC had a moderate reclassification rate $(2.4 \%)$. However, 6 of the 63 in symptomatic test cohorts assigned to another risk stratification. The patients we included were symptomatic and with relatively high Agatston calcification score (the median value was 199), the overall reclassification rate was 5.6\%. Only one patient changed from nonzero calcium score to zero calcium in ASIR-V 50\%, medium and high-level DLIR reconstruction. the use of DLIR-L did not increase the number of patients with zero CAC scores, so it is possible to be used in CAC scanning.

However, Gebhard et al [24] demonstrated that compared with FBP, reconstruction of increasing ASIR levels significantly reduced Agatston and volume score, whereas CAC mass remained unchanged. They hypothesized that IR reconstructions make the borders of the lesion smoother and the center appears denser. contrary to the results observed with ASIR, the decrease in CAC mass score with DLIR and ASIR-V were similar to Agatston score and CAC volume in our study, a small but significant reduction in mean mass scores were found. Therefore, before introducing the new reconstruction algorithm into clinical practice, these ambiguous results prompt us to adopt these techniques cautiously, standardization process in different vendors or reconstructionbased adjustment of CAC values are needed to minimize the influence of reconstruction algorithm on cardiovascular risk stratification.

At present, the disadvantage of mainstream iterative reconstruction is the change of image texture. Due to the different noise power spectrum of FBP and IR, many studies [12-13] have reported that with the intensification of iterative strength, "blotchy", "plastic-looking" or "unnatural" noise texture come out in the image, which may hinder the interpretation for the image. As a branch of machine learning and AI, deep learning overcomes the modeling limitations of IR by handling complex models and thousands of parameters in the training process [16]. DLIR uses the deep neural network (DNNs) trained with the FBP dataset of high-quality to 
generate images that accurately match the ground truth data, and retains the noise texture similar to high-dose FBP images [27]. With the increment of the reconstruction strength, the noise decreases and the signal-to-noise ratio increases significantly. recent published comparison studies [28, 29] between the same DLIR algorithm and IR in chest plain scan and abdominal multi-phase enhanced scan have proved that DLIR may help reduce the radiation exposure, and the noise level remains no change or significantly decreased.

To the best of our knowledge, there has not been reported on the effect of DLIR on cardiovascular risk restratification based on CAC score. In this study, the difference of calcification score among reconstructions were relatively small. Based on Bland-Altman analysis, the Agatston scores of ASIR-V and incremental DLIR levels as compared to FBP reconstruction have good agreement, there were no systematic bias. We confirm that the use of ASIR-V and DLIR significantly reduce noise in comparison to FBP, while hardly affect the Agatston score-based risk assessment and Agatston, age, sex, and race adjusted CAC percentile risk categories.

Our research has several limitations. First of all, this is a single-center study, we only investigated the effect of specific reconstruction algorithms from one CT vendor. Second, all images were post-processed by one observer in the same workstation to reduce the potential deviation of CACS measurement. Third, we did not prospectively apply low tube voltage / tube current techniques combined with DLIR in comparison with FBP in standard doses. As concerns about radiation exposure continue increasing, evaluating the potential of the novel reconstruction technology in the reduction of radiation exposure under the premise of without affecting calcium quantification should be considered, which is worthy of further study. Finally, our research included symptomatic patients predominantly at moderate risk, the median Agatston score was 199, only 7 patients with zero calcification, which potentially limited the applicability of our research. however, the inclusion of a large number of patients without coronary artery calcification will only add limited information to CAC comparability.

In conclusion, compared with the traditional FBP, the utilization of DLIR and ASIR-V reduce the coronary artery calcification score. however, the cardiac risk categorizations are comparable. As the advanced reconstruction method, DLIR and ASIR-V shows great potential in improving CT image quality. DLIR-L has the least effect on coronary calcium quantification compared to ASIR-V and DLIR at mediate and high levels. It may be considered as an alternative to FBP for routine clinical use. Further prospective research is needed to validate the influence of new reconstruction algorithms in large-scale patient groups with different risk characteristics.

\section{References}

[1] Detrano Robert, Guerci Alan D, Carr J Jeffrey et al (2008) Coronary calcium as a predictor of coronary events in four racial or ethnic groups. N Engl J Med 358(13):1336-1345.

https://doi.org/10.1056/NEJMoa072100

[2] Hecht HS (2015) Coronary artery calcium scanning: past, present, and future. JACC Cardiovasc Imaging. 8:579-596. https://doi.org/10.1016/j.jcmg.2015.02.006

[3] Greenland Philip, Blaha Michael J, Budoff Matthew J, Erbel Raimund, Watson Karol E (2018) Coronary Calcium Score and Cardiovascular Risk. J Am Coll Cardiol 72(4): 434-447. https://doi.org/10.1016/j.jacc.2018.05.027

[4] Tesche Christian, Duguay Taylor M, Schoepf U Joseph et al (2018). Current and future applications of CT coronary calcium assessment. Expert Rev Cardiovasc Ther 16(6): 441-453. https://doi.org/10.1080/14779072.2018.1474347

[5] Sulaiman Nada, Soon Jeanette, Park Jong Kwan et al (2017). Comparison of low-dose coronary artery calcium scoring using low tube current technique and hybrid iterative reconstruction vs. filtered back 
projection. Clin Imaging 43(undefined): 19-23. https://doi.org/10.1016/j.clinimag.2017.01.017

[6] Hecht Harvey S, Cronin Paul, Blaha Michael J, Budoff Matthew J, Kazerooni Ella A, Narula Jagat, Yankelevitz David, Abbara Suhny(2017) 2016 SCCT/STR guidelines for coronary artery calcium scoring of noncontrast noncardiac chest CT scans: A report of the Society of Cardiovascular Computed Tomography and Society of Thoracic Radiology. J Thorac Imaging, 32(5): W54-W66. https://doi.org/10.1097/RTI.0000000000000287

[7] Messenger Bradley, Li Dong, Nasir Khurram, Carr J Jeffrey, Blankstein Ron, Budoff Matthew J (2016) Coronary calcium scans and radiation exposure in the multi-ethnic study of atherosclerosis. Int J Cardiovasc Imaging 32(3): 525-529. https://doi.org/10.1007/s10554-015-0799-3

[8] Tatsugami Fuminari, Higaki Toru, Fukumoto Wataru, Kaichi Yoko, Fujioka Chikako, Kiguchi Masao, Yamamoto Hideya, Kihara Yasuki, Awai Kazuo (2015) Radiation dose reduction for coronary artery calcium scoring at 320-detector CT with adaptive iterative dose reduction 3D. Int J Cardiovasc Imaging 31(5): 10451052. https://doi.org/10.1007/s10554-015-0637-7

[9] Halliburton Sandra S, Tanabe Yuki, Partovi Sasan, Rajiah Prabhakar (2017) The role of advanced reconstruction algorithms in cardiac CT. Cardiovasc Diagn Ther 7(5): 527-538.

https://doi.org/10.21037/cdt.2017.08.12

[10] Pontone Gianluca, Muscogiuri Giuseppe, Andreini Daniele et al (2018) Impact of a New Adaptive Statistical Iterative Reconstruction (ASIR)-V Algorithm on Image Quality in Coronary Computed Tomography Angiography. Acad Radiol 25(10): 1305-1313. https://doi.org/10.1016/j.acra.2018.02.009

[11] Benz Dominik C, Gräni Christoph, Mikulicic Fran et al (2016). Adaptive Statistical Iterative Reconstruction-V: Impact on Image Quality in Ultralow-Dose Coronary Computed Tomography Angiography. J Comput Assist Tomogr, 40(6): 958-963. https://doi.org/10.1097/RCT.0000000000000460

[12] Benz Dominik C, Gräni Christoph, Mikulicic Fran et al (2016). Adaptive Statistical Iterative Reconstruction-V: Impact on Image Quality in Ultralow-Dose Coronary Computed Tomography Angiography. J Comput Assist Tomogr 40(6): 958-963. https://doi.org/10.1097/RCT.0000000000000460

[13] Samei Ehsan, Richard Samuel (2015) Assessment of the dose reduction potential of a model-based iterative reconstruction algorithm using a task-based performance metrology. Med Phys 42(1): 314-323. https://doi.org/10.1118/1.4903899

[14] Lin Andrew, Kolossváry Márton, Motwani Manish, Išgum Ivana, Maurovich-Horvat Pál, Slomka Piotr J, Dey Damini(2021) Artificial Intelligence in Cardiovascular Imaging for Risk Stratification in Coronary Artery Disease. Radiol Cardiothorac Imaging 3(1): e200512. https://doi.org/10.1148/ryct.2021200512

[15] Liu Peijun, Wang Man, Wang Yining et al (2020). Impact of Deep Learning-based Optimization Algorithm on Image Quality of Low-dose Coronary CT Angiography with Noise Reduction: A Prospective Study. Acad Radiol 27(9): 1241-1248. https://doi.org/10.1016/j.acra.2019.11.010

[16] Hsieh J, Liu E, Nett B, Tang J, Thibault J, Sahney S. A New Era of Image Reconstruction: TrueFidelity. White Paper (JB68676XX). GE Healthcare; 2019.

[17] Greffier Joël, Hamard Aymeric, Pereira Fabricio, Barrau Corinne, Pasquier Hugo, Beregi Jean Paul, Frandon Julien (2020) Image quality and dose reduction opportunity of deep learning image reconstruction algorithm for CT: a phantom study. Eur Radiol 30(7): 3951-3959. https://doi.org/10.1007/s00330-020-06724-w

[18] Benz Dominik C, Benetos Georgios, Rampidis Georgios et al (2020). Validation of deep-learning image reconstruction for coronary computed tomography angiography: Impact on noise, image quality and diagnostic 
accuracy. J Cardiovasc Comput Tomogr 14(5): 444-451. https://doi.org/10.1016/j.jcct.2020.01.002

[19] Akagi Motonori, Nakamura Yuko, Higaki Toru, Narita Keigo, Honda Yukiko, Zhou Jian, Yu Zhou, Akino Naruomi, Awai Kazuo (2019) Deep learning reconstruction improves image quality of abdominal ultra-highresolution CT. Eur Radiol 29(11): 6163-6171. https://doi.org/10.1007/s00330-019-06170-3

[20] McClelland Robyn L, Chung Hyoju, Detrano Robert, Post Wendy, Kronmal Richard A (2006) Distribution of coronary artery calcium by race, gender, and age: results from the Multi-Ethnic Study of Atherosclerosis (MESA). Circulation 113(1): 30-7. https://doi.org/10.1161/CIRCULATIONAHA.105.580696

[21] Kurata Akira, Dharampal Anoeshka, Dedic Admir, de Feyter Pim J, Krestin Gabriel P, Dijkshoorn Marcel L, Nieman Koen (2013) Impact of iterative reconstruction on CT coronary calcium quantification. Eur Radiol 23(12): 3246-52. https://doi.org/10.1007/s00330-013-3022-8

[22] Messerli Michael, Rengier Fabian, Desbiolles Lotus et al (2016) Impact of Advanced Modeled Iterative Reconstruction on Coronary Artery Calcium Quantification. Acad Radiol 23(12): 1506-1512. https://doi.org/10.1016/j.acra.2016.08.008

[23] Szilveszter Bálint, Elzomor Hesham, Károlyi Mihály et al (2016) The effect of iterative model reconstruction on coronary artery calcium quantification. Int J Cardiovasc Imaging 32(1): 153-160. https://doi.org/10.1007/s10554-015-0740-9

[24] Gebhard Cathérine, Fiechter Michael, Fuchs Tobias A et al (2013) Coronary artery calcium scoring: Influence of adaptive statistical iterative reconstruction using 64-MDCT. Int J Cardiol 167(6): 2932-2937. https://doi.org/10.1016/j.ijcard.2012.08.003

[25] Hecht Harvey S, Blaha Michael J, Kazerooni Ella A, Cury Ricardo C, Budoff Matt, Leipsic Jonathon, Shaw Leslee (2018) CAC-DRS: Coronary Artery Calcium Data and Reporting System. An expert consensus document of the Society of Cardiovascular Computed Tomography (SCCT). J Cardiovasc Comput Tomogr 12(3): 185-191. https://doi.org/10.1016/j.jcct.2018.03.008

[26] Oudkerk Matthijs, Stillman Arthur E, Halliburton Sandra S et al (2008) Coronary artery calcium screening: current status and recommendations from the European Society of Cardiac Radiology and North American Society for Cardiovascular Imaging. Int J Cardiovasc Imaging 24(6): 645-71. https://doi.org/10.1007/s10554008-9319-z

[27] Chartrand Gabriel, Cheng Phillip M, Vorontsov Eugene, Drozdzal Michal, Turcotte Simon, Pal Christopher J, Kadoury Samuel, Tang An (2017) Deep Learning: A Primer for Radiologists. Radiographics 37(7): 2113-2131. https://doi.org/10.1148/rg.2017170077

[28] Kim Joo Hee, Yoon Hyun Jung, Lee Eunju, Kim Injoong, Cha Yoon Ki, Bak So Hyeon (2021) Validation of Deep-Learning Image Reconstruction for Low-Dose Chest Computed Tomography Scan: Emphasis on Image Quality and Noise. Korean J Radiol 22(1): 131-138. https://doi.org/10.3348/kjr.2020.0116

[29] Cao Le, Liu Xiang, Li Jianying et al (2021) A study of using a deep learning image reconstruction to improve the image quality of extremely low-dose contrast-enhanced abdominal CT for patients with hepatic lesions. Br J Radiol 94(1118): 20201086. https://doi.org/10.1259/bjr.20201086 


\section{Tables}

Table 1 Baseline Characteristics and Radiological Parameters

\begin{tabular}{ll}
\hline Characteristics & Values \\
\hline Number of patients & 96 \\
Age $(\mathrm{y})$, mean $\pm \mathrm{SD}$ & $65.3 \pm 10.2$ \\
Male, $\mathrm{n}(\%)$ & $58(60)$ \\
Body mass index $\left(\mathrm{kg} / \mathrm{m}^{2}\right)$, mean $\pm \mathrm{SD}$ & $23.9 \pm 3.3$ \\
Hypertension, $\mathrm{n}(\%)$ & $37(39)$ \\
Smoker, $\mathrm{n}(\%)$ & $19(20)$ \\
Dyslipidemia, $\mathrm{n}(\%)$ & $53(55)$ \\
Diabetes, $\mathrm{n}(\%)$ & $26(27)$ \\
Mean heart rate $(\mathrm{bpm})$, mean $\pm \mathrm{SD}$ & $64.3 \pm 9.5$ \\
CTDI vol $(\mathrm{mGy})$, mean $\pm \mathrm{SD}$ & $4.8 \pm 0.2$ \\
DLP $\left(\mathrm{mGy} * \mathrm{~cm}{ }^{-1}\right)$, mean $\pm \mathrm{SD}$ & $68.3 \pm 3.4$ \\
ED $(\mathrm{mSv}), \mathrm{mean}^{\mathrm{S}} \mathrm{SD}$ & $0.93 \pm 0.15$ \\
\hline
\end{tabular}

bpm, beats per minute; CTDI vol, the volume CT dose index; DLP, doselength product, $\mathrm{ED}$, effective radiation dose. 
Table 2 Quantitative Image Analysis of Image Noise, SNR for reconstruction groups

\begin{tabular}{|c|c|c|c|c|}
\hline \multirow{2}{*}{ Reconstruction } & \multicolumn{4}{|c|}{ Quantitative image quality metrics (\%) } \\
\hline & Noise AR (HU) & $\begin{array}{l}\text { Signal-to-Noise } \\
\text { Ratio in AR }\end{array}$ & Noise LV (HU) & $\begin{array}{l}\text { Signal-to-Noise } \\
\text { Ratio in LV }\end{array}$ \\
\hline ASiR-V 50\% vs FBP & $-26.7 \pm 3.4^{*}$ & $36.5 \pm 5.8^{*}$ & $-25.9 \pm 3.2^{*}$ & $35 \pm 5.5^{*}$ \\
\hline DLIR-L vs FBP & $-27.6 \pm 8.3^{*}$ & $39.9 \pm 16.1^{*}$ & $-23.7 \pm 9.2 *$ & $32.1 \pm 18.3^{*}$ \\
\hline DLIR-M vs FBP & $-37.3 \pm 7.2^{*}$ & $61.3 \pm 17.7^{*}$ & $-34.6 \pm 7.7^{*}$ & $54.3 \pm 18.4^{*}$ \\
\hline DLIR-H vs FBP & $-46.4 \pm 5.4^{*}$ & $88.2 \pm 21.7^{*}$ & $-44.9 \pm 5.7^{*}$ & $82.5 \pm 19.9^{*}$ \\
\hline DLIR-L vs ASiR-V 50\% & $-1.4 \pm 9.7$ & $2.4 \pm 10.1$ & $2.9 \pm 10.9$ & $-2.3 \pm 11.6$ \\
\hline DLIR-M vs ASiR-V 50\% & $-14.6 \pm 8.7^{*}$ & $18.1 \pm 11.4^{*}$ & $-11.8 \pm 8.5^{*}$ & $14.1 \pm 11.7^{*}$ \\
\hline DLIR-H vs ASiR-V 50\% & $-26.9 \pm 6.4^{*}$ & $37.8 \pm 13.5^{*}$ & $-25.7 \pm 6.4^{*}$ & $35.1 \pm 11.2^{*}$ \\
\hline
\end{tabular}

Data given is mean relative differences \pm SD (expressed in percentages) between the targeted and the referencec reconstruction groups. $\mathrm{AR}=$ Aorta root, $\mathrm{LV}=$ left ventricular cavity; FBP $=$ filtered back projection; ASIR-V $50 \%=$ adaptive statistical iterative reconstruction-V at 50\% intensity; DLIR-L = deep learning image reconstruction at low level: DLIR-M = deep learning image reconstruction at medium level; DLIR-H = deep learning image reconstruction at high level. $\mathrm{HU}=$ Hounsfield units. ${ }^{*} p<0.05$. 
Table 3 Agatston, volumetric, mass scores with different reconstruction techniques

\begin{tabular}{ccccccc}
\hline & & FBP & ASIR-V & DLIR-L & DLIR-M & DLIR-H \\
\hline \multirow{2}{*}{ Agatston score } & Mean & $328.8 \pm 416.1$ & $314.3 \pm 407.2$ & $321.9 \pm 410.9$ & $315.6 \pm 402.1$ & $307.5 \pm 392.8$ \\
& Median & $199(32,451)$ & $188(26,422)$ & $196(30,426)$ & $192(28,419)$ & $185(28,408)$ \\
& Mean & $132.8 \pm 158.9$ & $126.3 \pm 154.8$ & $122.6 \pm 149.6$ & $119.6 \pm 146.7$ & $116.8 \pm 143.1$ \\
$\begin{array}{c}\text { Volumetric score } \\
\left(\mathrm{mm}^{3}\right)\end{array}$ & Median & $99(20.8,179.5)$ & $95(15,165)$ & $95.5(15.8,173)$ & $93(15,169.8)$ & $91(14,164.8)$ \\
& Mean & $52.9 \pm 72.8$ & $51.3 \pm 71.7$ & $52.3 \pm 72.8$ & $51.6 \pm 71.6$ & $51.0 \pm 70.9$ \\
Mass score $(\mathrm{mg})$ & Median & $32(7,63)$ & $29(6,61)$ & $31(6.5,63)$ & $30(6.5,59)$ & $30(6.5,59)$ \\
\hline
\end{tabular}

Data given is mean \pm SD or IQR. FBP $=$ filtered back projection; ASIR-V $50 \%=$ adaptive statistical iterative reconstruction$\mathrm{V}$ at $50 \%$ intensity; DLIR-L = deep learning image reconstruction at low level; DLIR-M = deep learning image reconstruction at medium level; DLIR-H = deep learning image reconstruction at high level. 


\section{Figure captions}

Fig. 1 Box-and-whisker plots of quantitative comparisons among FBP, ASIR-V, and three levels of DLIR, including image noise in aortic root (a) and left ventricular cavity (b), and SNR in aortic root (c) and left ventricular cavity (d). The figure shows the data of median, interquartile range (IQR) range between the 25th and 75th percentiles (box), and 1.5 IQR range (whisker). FBP = filtered back projection; ASIR-V = Adaptive Statistical Iterative Reconstruction-V; DLIR-L, DLIR-M, and DLIR-H = deep learning image reconstruction at low, medium, and high levels

Fig. 2 Axial cardiac CT images of a 75-year-old man (body mass index, $26.5 \mathrm{~kg} / \mathrm{m}^{2}$ ) reconstructed with FBP, ASIR-V 50\% and three levels of DLIR (DLIR-L, M, and H, respectively), The section thickness was $2.5 \mathrm{~mm}$. Image noise was 31.1 HU, 23 HU, 26.2 HU, 20.9 HU, and 18.1 HU in the left ventricular. At the level of the distal segment of the right coronary artery (RCA) artery, the dots marked manually by red color were calcified lesions. There are scattered false-positive dots (marked by green color automatically) on FBP (a), ASIR-V 50\% (b) and DLIR with incremental strength levels (c, d, e) images. One calcified plaque could not detect in ASIRV 50\% group (white arrows)

Fig. 3 Bland-Altman plots of all measured Agatston scores of ASIR-V 50\% and three levels of DLIR (DLIR-L, $\mathrm{M}$, and $\mathrm{H}$, respectively) comparing with FBP. The limit of agreement and mean difference for the Agatston scores were shown in a-d, respectively 


\section{Figures}

Fig. 1

(a)

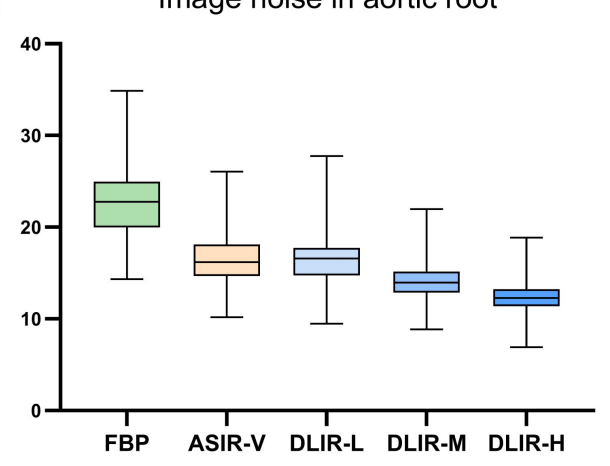

(c)

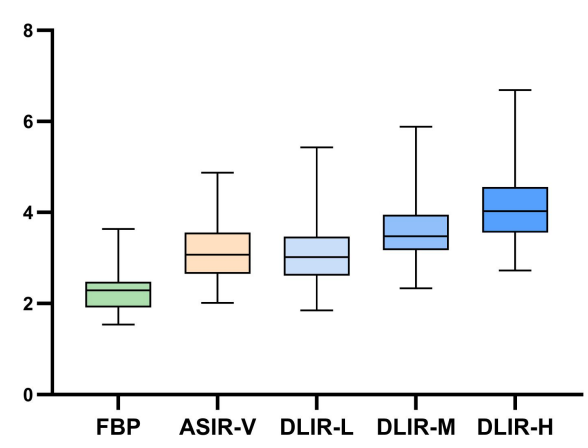

(b)

Image noise in left ventricular cavity

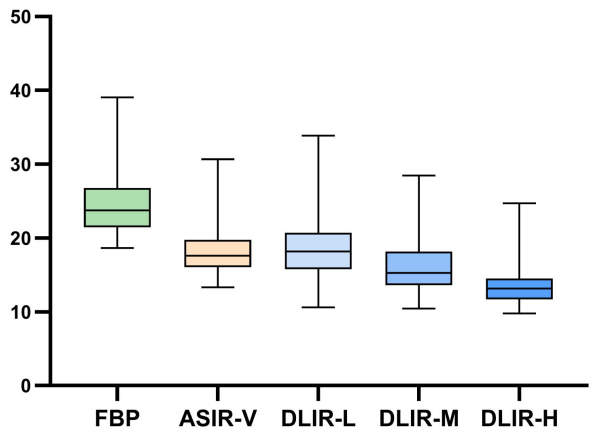

(d)

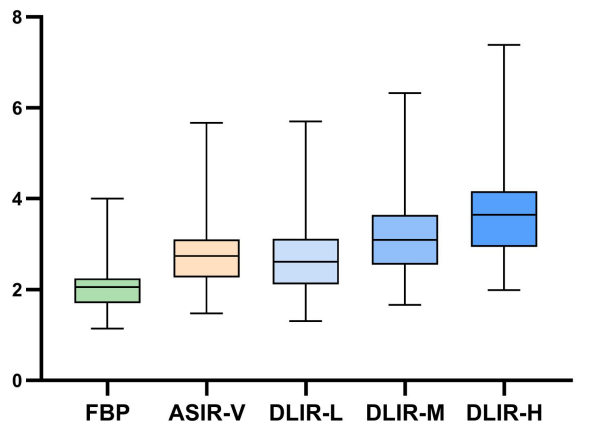


Fig. 2

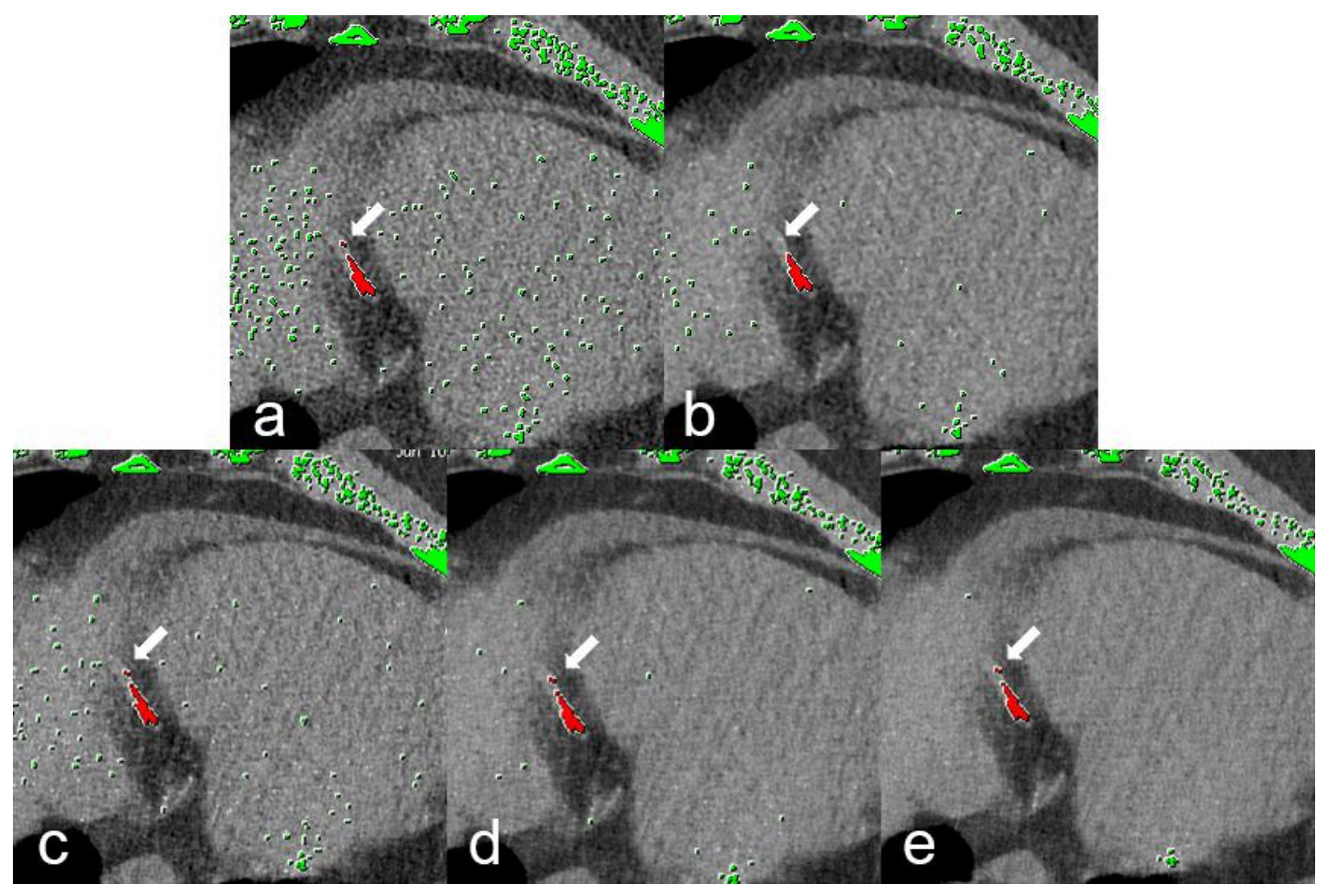


Fig. 3

Agatston score
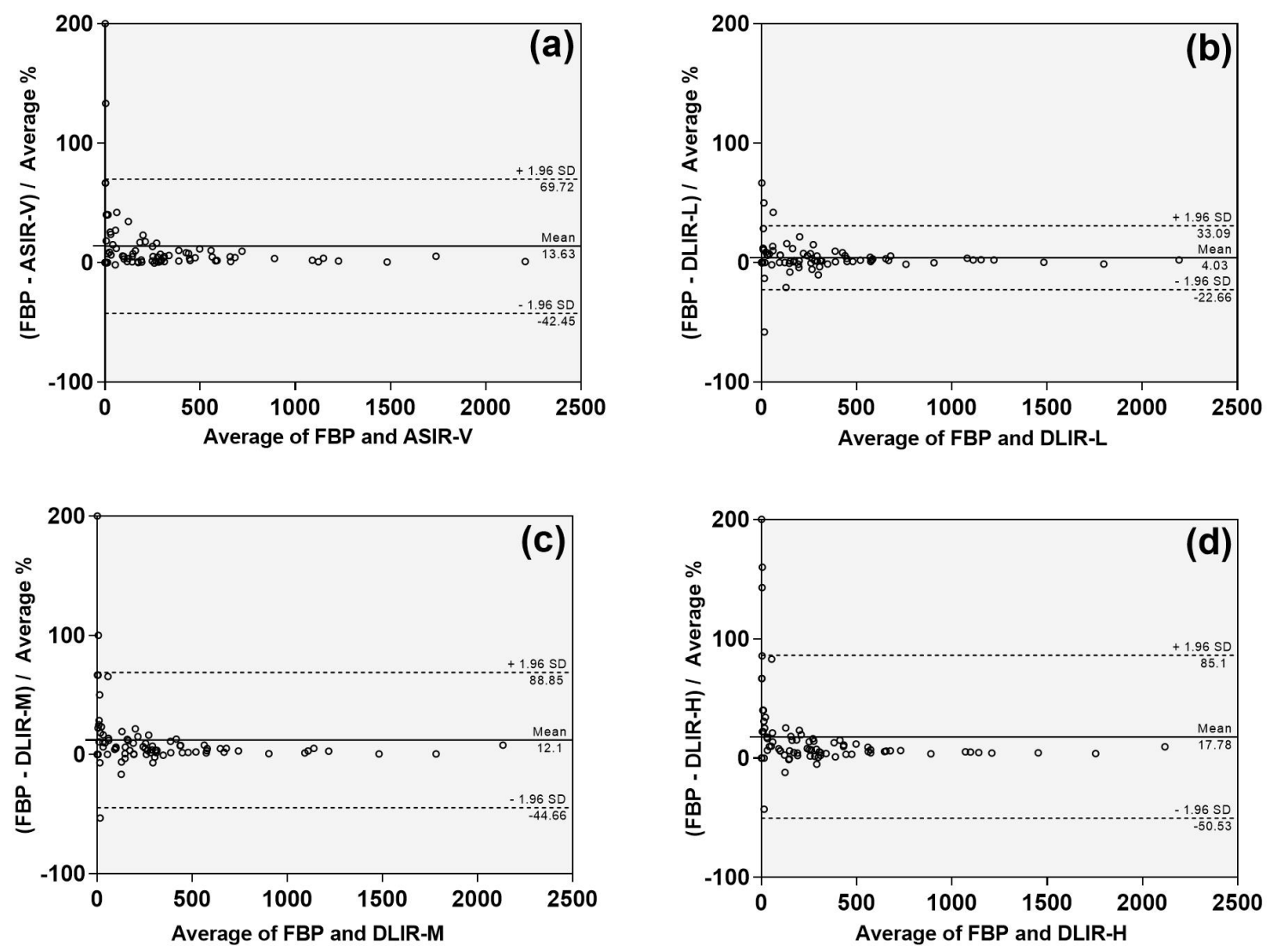
Online Resource 1 Quantitative image analysis of CT value, image noise, and SNR for five reconstruction groups

\begin{tabular}{|c|c|c|c|c|c|c|}
\hline \multirow{2}{*}{ Reconstruction } & \multicolumn{6}{|c|}{ Quantitative image quality metrics } \\
\hline & Signal AR (HU) & Noise AR (HU) & $\begin{array}{c}\text { Signal-to-Noise } \\
\text { Ratio in AR }\end{array}$ & Signal LV (HU) & Noise LV (HU) & $\begin{array}{l}\text { Signal-to-Noise } \\
\text { Ratio in LV }\end{array}$ \\
\hline FBP & $50.8 \pm 8.7$ & $22.9 \pm 4.6$ & $2.3 \pm 0.5$ & $48.4 \pm 8.3$ & $24.7 \pm 4.8$ & $2.0 \pm 0.6$ \\
\hline ASIR-V 50\% & $50.7 \pm 8.5$ & $16.8 \pm 3.6$ & $3.1 \pm 0.7$ & $48.3 \pm 8.3$ & $18.3 \pm 3.9$ & $2.8 \pm 0.8$ \\
\hline DLIR-L & $50.8 \pm 8.7$ & $16.5 \pm 3.8$ & $3.2 \pm 0.8$ & $48.2 \pm 8.4$ & $18.9 \pm 4.9$ & $2.7 \pm 0.9$ \\
\hline DLIR-M & $50.7 \pm 8.6$ & $14.2 \pm 2.7$ & $3.7 \pm 0.8$ & $48.2 \pm 8.4$ & $16.1 \pm 3.7$ & $3.2 \pm 1.0$ \\
\hline DLIR-H & $50.7 \pm 8.5$ & $12.3 \pm 2.6$ & $4.3 \pm 1.1$ & $48.1 \pm 8.5$ & $13.6 \pm 3.2$ & $3.7 \pm 1.2$ \\
\hline
\end{tabular}

Data given is mean $\pm \mathrm{SD} . \mathrm{AR}=$ Aorta root, $\mathrm{LV}=$ left ventricular cavity; FBP $=$ filtered back projection; ASIR-V $50 \%=$ adaptive statistical iterative reconstruction-V at 50\% intensity; DLIR-L = deep learning image reconstruction at low level; DLIR-M = deep learning image reconstruction at medium level; DLIR-H $=$ deep learning image reconstruction at high level. $\mathrm{HU}=$ Hounsfield units. 
Online Resource 2 Influence of ASIR-V 50\%, DLIR-L, DLIR-M and DLIR-H on Agatston score-based risk categories

\begin{tabular}{|c|c|c|c|c|c|c|c|c|c|c|c|c|c|c|c|c|c|c|c|c|}
\hline \multirow{3}{*}{$\begin{array}{l}\text { Severity } \\
\text { class with } \\
\text { FBP }\end{array}$} & \multicolumn{20}{|c|}{ Severity classification with ASIR-V and DLIR } \\
\hline & \multicolumn{5}{|c|}{ ASIR-V 50\% } & \multicolumn{5}{|c|}{ DLIR-L } & \multicolumn{5}{|c|}{ DLIR-M } & \multicolumn{5}{|c|}{ DLIR-H } \\
\hline & $\begin{array}{c}\text { no } \\
\text { risk }\end{array}$ & low & mod. & high & $\begin{array}{l}\text { very } \\
\text { high }\end{array}$ & $\begin{array}{c}\text { no } \\
\text { risk }\end{array}$ & low & mod. & high & $\begin{array}{l}\text { very } \\
\text { high }\end{array}$ & $\begin{array}{l}\text { no } \\
\text { risk }\end{array}$ & low & mod. & high & $\begin{array}{l}\text { very } \\
\text { high }\end{array}$ & $\begin{array}{c}\text { no } \\
\text { risk }\end{array}$ & low & mod. & high & $\begin{array}{l}\text { very } \\
\text { high }\end{array}$ \\
\hline $\begin{array}{l}\text { no risk } \\
(\mathrm{n}=7)\end{array}$ & 7 & 0 & 0 & 0 & 0 & 7 & 0 & 0 & 0 & 0 & 7 & 0 & 0 & 0 & 0 & 7 & 0 & 0 & 0 & 0 \\
\hline $\begin{array}{c}\text { low } \\
(\mathrm{n}=12)\end{array}$ & $1 *$ & 11 & 0 & 0 & 0 & 0 & 12 & 0 & 0 & 0 & $1 *$ & 11 & 0 & 0 & 0 & $1 *$ & 11 & 0 & 0 & 0 \\
\hline $\begin{array}{c}\text { mod. } \\
(n=15)\end{array}$ & 0 & $2 *$ & 13 & 0 & 0 & 0 & $2 *$ & 13 & 0 & 0 & 0 & $2 *$ & 13 & 0 & 0 & 0 & $2 *$ & 13 & 0 & 0 \\
\hline $\begin{array}{c}\text { high } \\
(\mathrm{n}=32)\end{array}$ & 0 & 0 & $1 *$ & 31 & 0 & 0 & 0 & $1 *$ & 31 & 0 & 0 & 0 & $1 *$ & 31 & 0 & 0 & 0 & $1 *$ & 31 & 0 \\
\hline $\begin{array}{c}\text { Total } \\
(\mathrm{n}=96)\end{array}$ & 8 & 13 & 14 & 32 & 29 & 7 & 14 & 14 & 31 & 30 & 8 & 13 & 14 & 32 & 29 & 8 & 13 & 14 & 32 & 29 \\
\hline
\end{tabular}

* Numbers indicate patients who changed severity classification with use of ASIR-V and DLIR. mod. = moderate. ASIR-V 50\% $=$ adaptive statistical iterative reconstruction Veo at 50\% intensity, DLIR-L, DLIR-M, and DLIR-H = deep learning image reconstruction at low, medium, and high levels. 
Online Resource 3 Influence of ASIR-V 50\%, DLIR-L, DLIR-M and DLIR-H on CAC percentile risk categories adjusted by Agatston score, age, gender and race

\begin{tabular}{|c|c|c|c|c|c|c|c|c|c|c|c|c|c|c|c|c|c|c|c|c|}
\hline \multirow{3}{*}{$\begin{array}{c}\text { Severity } \\
\text { class } \\
\text { with FBP } \\
\text { (th) }\end{array}$} & \multicolumn{20}{|c|}{ Severity classification with ASIR-V and DLIR (th) } \\
\hline & \multicolumn{5}{|c|}{ ASIR-V 50\% } & \multicolumn{5}{|c|}{ DLIR-L } & \multicolumn{5}{|c|}{ DLIR-M } & \multicolumn{5}{|c|}{ DLIR-H } \\
\hline & $<25$ & $26-50$ & $51-75$ & $76-90$ & $>90$ & $<25$ & $26-50$ & $51-75$ & $76-90$ & $>90$ & $<25$ & $26-50$ & $51-75$ & $76-90$ & $>90$ & $<25$ & $26-50$ & $51-75$ & $76-90$ & $>90$ \\
\hline $\begin{array}{c}<25 \\
(n=9)\end{array}$ & 9 & 0 & 0 & 0 & 0 & 9 & 0 & 0 & 0 & 0 & 9 & 0 & 0 & 0 & 0 & 9 & 0 & 0 & 0 & 0 \\
\hline $\begin{array}{l}26-50 \\
(n=7)\end{array}$ & 0 & 7 & 0 & 0 & 0 & 0 & 7 & 0 & 0 & 0 & 0 & 7 & 0 & 0 & 0 & 0 & 7 & 0 & 0 & 0 \\
\hline $\begin{array}{c}51-75 \\
(n=17)\end{array}$ & $1 *$ & 0 & 16 & 0 & 0 & 0 & 0 & 17 & 0 & 0 & $1 *$ & 0 & 16 & 0 & 0 & $1 *$ & $1 *$ & 15 & 0 & 0 \\
\hline $\begin{array}{c}76-90 \\
(n=27)\end{array}$ & 0 & 0 & $2 *$ & 25 & 0 & 0 & 0 & $2 *$ & 25 & 0 & 0 & 0 & $2 *$ & 25 & 0 & 0 & 0 & $2 *$ & 25 & 0 \\
\hline $\begin{array}{c}>90 \\
(n=25)\end{array}$ & 0 & 0 & 0 & $1 *$ & 24 & 0 & 0 & 0 & $1 *$ & 24 & 0 & 0 & 0 & $1 *$ & 24 & 0 & 0 & 0 & $1 *$ & 24 \\
\hline $\begin{array}{c}\text { Total } \\
(\mathrm{n}=89)\end{array}$ & 10 & 7 & 18 & 26 & 24 & 9 & 7 & 19 & 26 & 24 & 10 & 7 & 18 & 26 & 24 & 10 & 8 & 17 & 26 & 24 \\
\hline
\end{tabular}

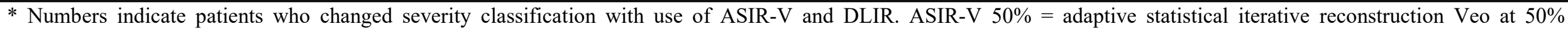
intensity, DLIR-L, DLIR-M, and DLIR-H = deep learning image reconstruction at low, medium, and high levels. 


\section{Supplementary Files}

This is a list of supplementary files associated with this preprint. Click to download.

- 4.SupplementaryInformation.21.07.27.pdf 